\title{
Importance of ADME and Bioanalysis in the Drug Discovery
}

\author{
Pradeep K Vuppala $^{1 *}$, Dileep R Janagam ${ }^{2}$ and Pavan Balabathula ${ }^{2}$
}

${ }^{1}$ Preclinical Pharmacokinetics Shared Resource, St. Jude Children's Research Hospital, Memphis, TN, USA

${ }^{2}$ University of Tennessee Health Sciences Center, Memphis, TN, USA

\section{Editorial}

The hunt for new drugs can be divided into two stages: discovery and development. Drug discovery includes generating a hypothesis of the target receptor for a particular disorder and screening the in vitro and/or in vivo biological activities of the new drug candidates. Drug development involves the assessment of efficacy and toxicity of the new drug candidates.

To aid in a discovery program, accurate data on pharmacokinetics and metabolism must be available as early as possible as it eventually contributes to the final success or failure of the compound. The initiation of early absorption, distribution, metabolism and excretion (ADME) screening has dramatically decreased the proportion of compounds failing in clinical trials. The main aim of preclinical ADME is to eliminate weak drug candidates in the early stages of drug development which allow resources to be focused on potential drug candidates [1]. Undesirable pharmacokinetic properties, such as poor absorption, too long or too short half-life $\left(t_{1 / 2}\right)$, and extensive first-pass metabolism majorly contribute to the failure of many drug candidates in early stages of drug development programs. To be successful, a drug candidate needs to posses good bioavailability and a desirable half life $\left(t_{1 / 2}\right)$. Early ADME provides the necessary data for selecting preclinical candidates, appropriate dosage forms, formualtion and accelerates the timeline for investigational new drug applications and subsequently new drug application submission to the FDA $[2,3]$.

Pharmacokinetic (PK) parameters are extrapolated from measurement of drug concentration in the plasma, blood, or other biological matrix over a selected time period. Pharmacokinetic data provides information that can guide future animal and clinical studies for the selection of the dose levels and frequency of administration. Many of the approved drugs generate metabolites which possess biological activity. These active metabolites may have different pharmacology and PK properties than the parent drug. A thorough understanding of the properties of active metabolite is important for estimating toxicity and therapeutic outcome. It is ideal to assess the metabolism of new drugs in vitro before proceeding to clinical studies. Early information about the enzymes involved in the drug metabolism is very useful in the design of drug-drug interactions studies $[4,5]$.

The prime determinant of efficacy and unexpected toxicity of a drug is how it penetrates biological barriers such as intestinal wall, or bloodbrain barrier (BBB). This is true in central nervous system (CNS) drugs, because drug candidates possessing in vitro efficacy do not penetrate the $\mathrm{BBB}$ will not show in vivo efficacy. The entry of drugs in to the CNS is limited by the presence of the BBB. BBB effectively isolates the brain from the blood because of the presence of tight junctions connecting the endothelial cells of the brain vessels. In addition to this, drug metabolizing enzymes and efflux pumps, such as $\mathrm{P}$-glycoprotein (P-gp) and the multi-drug-resistance protein located within the endothelial cells, push out the exogenous molecules from the brain. As a result, CNS drugs have high failure rate in the developmental stages [6].
Bioanalytical support plays a vital role during the lead optimization stages. The major goal of the bioanalysis is to assess the over-all ADME characteristics of the new chemical entities (NCE's). Arrays of bioanalytical methods are required to completely describe the pharmacokinetic behavior in laboratory animals as well as in humans [7]. Bioanalytical tools can play a significant role for the progress in drug discovery and development. Physiologic fluids such as blood, serum, plasma, urine and tissues are analyzed to determine the absorption and disposition of a drug candidate administered to a test animal [8]. Often the concentration of the NCE's in the biological matrix changes with time, and perhaps fall below nanogram level, therefore, quantification limits for the bioanalytical methods should be much lower than those required for analytical methods [9]. Effects from the endogenous materials (such as plasma proteins) of the biological matrix and stability issues make the accurate analysis difficult. Methods developed to analyze the pharmacokinetic study samples need complete separation of the analytes from matrix components. The performance of the bioanalytical assay can be improved by removing interferences from the matrix trough complex sample preparation steps, and concentrating the analyte of interest. Common methods of sample preparation include protein precipitation, liquid-liquid extraction and solid phase extraction [10]. Appropriate bioanalytical methods are required to detect drug candidate at low nanogram levels, at the same time linearly over three orders of magnitude [8].

\section{References}

1. Kaitin KI (2008) Obstacles and opportunities in new drug development. Clin Pharmacol Ther 83: 210-212.

2. Baumann A (2009) Nonclinical development of biopharmaceuticals. Drug Discov Today 14: 1112-1122.

3. Gannu R, Vuppala PK, Madishetty S, Ravula S, Yamsani MR (2010) Optimization of matrix diffusion mediated transdermal therapeutic system for buspirone: effect of variables on in vitro release, ex vivo permeation and pharmacotechnical properties. Acta Pharmaceutica Sinica 52: 315-327.

4. Roberts SA (2003) Drug metabolism and pharmacokinetics in drug discovery. Curr Opin Drug Discov Devel 6: 66-80.

5. el-Bacha RS, Minn A (1999) Drug metabolizing enzymes in cerebrovascular endothelial cells afford a metabolic protection to the brain. Cell Mol Biol (Noisyle-grand) 45: 15-23.

6. James ML, Shen B, Zavaleta CL, Nielsen CH, Mesangeau C, et al. (2012) New positron emission tomography (PET) radioligand for imaging II $f-1$ receptors in living subjects. J Med Chem 55: 8272-8282.

${ }^{*}$ Corresponding author: Pradeep K Vuppala, Preclinical Pharmacokinetics Shared Resource, St. Jude Children's Research Hospital, Memphis, TN, USA E-mail: pradeepkvuppala@gmail.com

Received June 03, 2013; Accepted June 05, 2013; Published June 10, 2013

Citation: Pradeep KV, Dileep RJ, Pavan B (2013) Importance of ADME and Bioanalysis in the Drug Discovery. J Bioequiv Availab 5: e31. doi:10.4172/ jbb.10000e31

Copyright: (c) 2013 Pradeep KV, et al.. This is an open-access article distributed under the terms of the Creative Commons Attribution License, which permits unrestricted use, distribution, and reproduction in any medium, provided the original author and source are credited. 
Citation: Pradeep KV, Dileep RJ, Pavan B (2013) Importance of ADME and Bioanalysis in the Drug Discovery. J Bioequiv Availab 5: e31. doi:10.4172/ jbb.10000e31

Page 2 of 2

7. Jamalapuram S, Vuppala PK, Mesangeau C, McCurdy CR, Avery BA (2012) Determination of a highly selective mixed-affinity sigma receptor ligand, in rat plasma by ultra performance liquid chromatography mass spectrometry and its application to a pharmacokinetic study. J Chromatogr B Analyt Technol Biomed Life Sci. 1: 1-6.

8. Jamalapuram S, Vuppala PK, Abdelazeem AH, McCurdy CR, Avery BA (2013) Ultra-performance liquid chromatography tandem mass spectrometry method for the determination of AZ66, a sigma receptor ligand, in rat plasma and its application to in vivo pharmacokinetics. Biomed Chromatogr.
9. Hsieh Y (2008) HPLC-MS/MS in drug metabolism and pharmacokinetic screening. Expert Opin Drug Metab Toxicol 4: 93-101.

10. Vuppala PK, Boddu SP, Furr EB, McCurdy CR, Avery BA (2011) Simple Sensitive, High-Throughput Method for the Quantification of Mitragynine in Rat Plasma Using UPLC-MS and Its Application to an Intravenous Pharmacokinetic Study. Chromatographia 74: 703-710. 